\title{
Octave equivalence as measured by similarity ratings
}

\author{
HOWARD J. KALLMAN \\ State University of New York, Albany, New York
}

\begin{abstract}
Octave equivalence refers to the musical equivalence of notes separated by one or more octaves; such notes are assigned the same name in the musical scale. The present series of experiments was an attempt to determine whether octave equivalence would be incorporated into subjects' similarity ratings of pairs of tones or tone sequences. In the first experiment, subjects on each trial rated the similarity of two successively presented tones. The results failed to show evidence of octave equivalence. In subsequent experiments, the range of frequency values presented and the musical context were manipulated. Evidence of octave equivalence was found only when the range of tone height differences presented was small; the effect of musical context was negligible. The implications of these results for theories of music perception and recognition are discussed.
\end{abstract}

Psychophysicists have developed unidimensional scales of pitch in which pitch sensation is viewed as a monotonic function of the frequency of presented tones (e.g., Stevens \& Galanter, 1957; Stevens \& Volkmann, 1940; Stevens, Volkmann, \& Newman, 1937). In contrast, theorists interested in musical aspects of tone perception suggest that an adequate scaling of pitch must encompass aspects of musicality. The present series of experiments was designed to evaluate one of the claims made by those who favor a musical interpretation of pitch, namely that under certain conditions, a musical phenomenon-octave equivalence-is necessary to describe the perceived similarity of tones or groups of tones.

Octave equivalence refers to the musical equivalence of notes that are separated in frequency by exactly one or more octaves (two notes are said to be separated by an octave if the ratio of their frequencies is $2: 1$ ). In the diatonic scales of western music, notes separated by one or more octaves are given the same note name, and, in fact, the central role of the octave in music has a long history (Révész, 1954) and is incorporated in most modern-day musical systems (Dowling, 1982). In recognition of the musical equivalence of notes that differ by one or more octaves, Révész (1954) referred to such notes as equiv-

Portions of this report are based on a PhD thesis submitted to the University of Wisconsin-Madison. I would like to thank Lola Lopes, Gregg Oden, Willard Thurlow, and especially the committee chairman, Dominic Massaro, for their contributions to the thesis. The research was supported by NIMH Grant MH36334 to Dominic W. Massaro, and preparation of this report was supported by NIMH Grant MH36455 to Howard J. Kallman. Reprint requests should be sent to Howard J. Kallman, Psychology Department, 1400 Washington Avenue, SUNY-Albany, Albany, New York 12222. alent notes and proposed a two-component theory of pitch in which not only tone height (which is a continuous dimension that increases with increases in tone frequency) but also tone quality, or synonymously tone chroma (i.e., the position of a note within the musical octave), was incorporated. ${ }^{1}$ To illustrate the distinction between tone height and chroma, consider two $A$ notes that have fundamental frequencies of 440 and $880 \mathrm{~Hz}$, respectively. In terms of tone height, the two notes would differ, but because they represent the same note name the two notes would be considered identical in terms of tone chroma.

Experimental evidence for the similarity of equivalent notes separated by one or more octaves was provided by conditioning studies that used both rats (Blackwell \& Schlosberg, 1943) and human subjects (Humphreys, 1939). These studies found greater generalization for tones an octave apart than for tones having frequencies somewhat less than an octave removed from the conditioned stimulus. The demonstration of octave generalization with rats suggests that octave effects may occur even in the absence of a culturally defined musical system of tonality.

Further support for the psychological similarity of octave-equivalent notes comes from work on tone memory interference. Deutsch (1973) found that tones that are octave equivalents have similar disruptive effects on memory of a to-be-remembered comparison tone. Finally, a number of recent studies have shown that listeners can generally identify melodies in which the sizes of the original melodic intervals are stretched by one or more octaves, although in some cases identification is somewhat worse than for the original undistorted melodies (Dowling \& Hollombe, 1977; Idson \& Massaro, 1978; Kallman \& Massaro, 1979; Massaro, Kallman, \& Kelly, 1980). 
That subjects can generally recognize octave-stretched melodies provides a strong demonstration of octave equivalence, because it shows that recognition is possible when octave-equivalent notes are substituted for the originals. ${ }^{2}$

Although a number of studies have provided clear evidence of octave equivalence, it is troubling that a few have not. Allen (1967) failed to find octaveequivalence effects when nonmusically trained subjects rated the similarities of pairs of tones, some of which were octave equivalents. In contrast, musically trained subjects rated tones that were separated by exactly one or more octaves as highly similar relative to other tone pairs. This difference between musical and nonmusical subjects could be explained perhaps by assuming that some aspect of musical training provided the musically trained subjects with a framework for interpreting the octave equivalents as highly similar to each other. And, in fact, Thurlow and Erchul (1977) proposed such an explanation to explain their failure to find consistent octave-equivalence effects in a series of experiments that included both interval identification and similarity rating tasks. But, given the body of evidence reviewed above that suggests that octave equivalence is a widespread phenomenon that can be found even in the generalization gradients of rats, it is unsatisfying to conclude that lack of musical training can explain specific failures to find evidence of octave equivalence.

The present series of experiments was an attempt to determine, under a variety of experimental conditions, whether subjects, when asked to directly rate the similarity of tones and tone sequences, would rate octave equivalents as perceptually similar. In light of the conflicting results of earlier experiments, many of which used rather indirect methods to tap octave equivalence, it was of interest to determine the conditions under which octave-equivalence effects would be found using a direct rating procedure.

Experiment 1 was designed to determine whether subjects' ratings of the perceived similarity of tones separated by one or more octaves would show octave equivalence. On each trial of the experiment, subjects heard two successive tones that were separated by a variable number of semitones. ${ }^{3}$ If octave equivalence obtained, it would have been reflected in an increase in the rated similarity of octave-equivalent tones relative to tones that were not octave equivalents but were separated by approximately the same number of semitones as were the octave-equivalent tones. ${ }^{4}$

\section{EXPERIMENT 1}

\section{Method}

Subjects. Thirty-five students at the University of Wisconsin with no history of hearing disorders volunteered to participate in one 1-h session to earn extra credit points in an introductory psychology course. In all of the present experiments, the subjects were tested in groups of four or fewer and were seated in individual sound-attenuated rooms throughout the experiment.

Stimuli and Procedure. The subject's task was to listen to two consecutively presented sine-wave tones and to rate the degree to which they were perceived as similar to each other. Each trial of the experiment began with the presentation of a visual warning signal for $750 \mathrm{msec}$. A 165 -msec sine-wave tone was presented $250 \mathrm{msec}$ after termination of the warning signal. Following presentation of the sine-wave tone, there was a 530 -msec silent interval, which was followed by presentation of a second 165-msec sine-wave tone. At termination of the second tone, a visual signal was illuminated indicating to subjects the beginning of the response period. Each subject made his/her response by setting the point of a linear potentiometer, $5.5 \mathrm{~cm}$ long, to a position corresponding to how similar the two tones sounded to each other. The leftmost portion was labeled "DIS"; the rightmost position was labeled "SIM." The subjects were told that the response scale was to be interpreted as a continuous one ranging from very dissimilar to very similar. Responses were to be made on the basis of the subject's intuitive feelings about how similar the two tones sounded to each other; the subjects were instructed not to "think too much" about their responses. When the subject was satisfied with his/her placement of the sliding pointer, s/he pressed a small button to the right of the sliding pointer, and the computer recorded the response.

The linear potentiometers on which the responses were recorded acted as voltage dividers. The resulting voltage from each potentiometer was measured by a multiplexed A-D converter and scaled so that the resulting score varied on an interval from 0 to 49 . When analyzing the data, this interval was normalized so that it varied between 0 and 1 .

The response period lasted until all subjects had registered their responses. The intertrial interval was $1 \mathrm{sec}$.

There were two within-subject variables in the experiment. The first was the frequency separation in semitones between the two tones presented on a given trial. The two tones were separated by $0,2,4,6,8,10,11,12,13,14,16,18,20,22,23,24,25,26$, or 28 semitones (19 levels). The second variable was whether the first or the second presented tone was the lower in frequency; this variable will be referred to as frequency direction. Frequency direction was a dummy variable under the 0-semitone level of frequency separation. The frequency of the lowest frequency tone on each trial was randomly chosen from the range $400-800 \mathrm{~Hz}$ with the restriction that the value was a multiple of 10 .

Each subject participated in 11 blocks of 38 trials each. Within each block, the experimental conditions occurred randomly without replacement. The first block was treated as practice to familiarize subjects both with the range of stimuli used and the task. Consequently, 10 blocks of trials were subjected to data analysis. Because each block contained one replication of the experimental conditions, each subject's data represented 10 replications.

The sine-wave tones were generated by a Wavetek Model 155 digitally controlled oscillator, and the output was gated by an Iconic Model 0137 computer-controlled audio switch to a McIntosh Model MC-50 amplifier. The tones were presented binaurally at approximately $76 \mathrm{~dB}(\mathrm{~A})$ through Grason-Stadler Model TDH-49 matched headphones. Tones began at the zero crossing, and rise and fall times were $25 \mathrm{msec}$. Although the Wavetek oscillator can be adjusted by units of $1 \mathrm{~Hz}$ when set to a value below $1,000 \mathrm{~Hz}$, it cannot be adjusted by units of less than $10 \mathrm{~Hz}$ when set to values above $1,000 \mathrm{~Hz}$. Thus, above $1,000 \mathrm{~Hz}$, the frequencies of the tones were rounded to the nearest 10 s place.

The visual displays were presented over Monsanto MDA-III light-emitting diodes. The visual warning signal consisted of an asterisk; the signal indicating the beginning of the response period was the letter " $R$."

\section{Results}

The data for each subject were averaged over the 10 experimental blocks prior to an analysis of vari- 
ance. Figure 1 presents the average similarity ratings as a function of the two tone's frequency separation in semitones and the direction of frequency change; a rating of " 1 " corresponds to a judgment that the two tones were very similar, whereas a rating of " 0 " corresponds to a judgment that they were very dissimilar.

The two main effects and their interaction were significant [frequency separation, $F(18,612)=222.99$, $\mathrm{MSe}=.011, \mathrm{p}<.01$; frequency direction, $\mathrm{F}(1,34)=$ $10.29, \mathrm{MSe}=.019, \mathrm{p}<.01$; frequency separation $\times$ frequency direction, $F(18,612)=6.10, M S e=.003$, $p<.01]$. The pattern of the similarity ratings are quite orderly: generally, the perceived similarity of the two presented tones decreased with increases in the number of semitones separating the two tones. Of particular interest, no evidence of octave equivalence was found in the group data. The data points representing one-octave (12-semitone) and two-octave 24-semitone) frequency separations follow the monotonic trend. Evidence for octave equivalence would have required that these points be elevated relative to those surrounding them.

Although the lack of octave equivalence is quite evident from an inspection of Figure 1, a trend analysis on selected data points was performed to confirm the absence of octave equivalence. The analysis involved summing for each subject the data points for (1) 11 and 23 semitones, (2) 12 and 24 semitones, and (3) 13 and 25 semitones. These sums will be referred to as Sums A, B, and C, respectively. Sum A was based on the ratings of tones that were separated by an interval that was one semitone smaller than would have been required for the tones to be octave equivalent. Sum B was based on the ratings of octaveequivalent tones. And Sum $C$ was based on the ratings of tones separated by an interval that was one semitone larger than that for octave-equivalent tones. The trend analysis was performed on the ordered set of sums: $A, B$, and $C$. If octave equivalence obtained, Sum B would have been elevated relative to $A$ and $C$, and consequently, a significant quadratic component would be found. In contrast, an absence of octave equivalence would suggest that most of the variance should be concentrated in the linear component because, on average, Sums A, B, and C represent, respectively, frequency separations of increasing size. In fact, the linear trend component was highly significant $[F(1,34)=37.44, p<.01]$, but the quadratic was not $(F<1)$.

At intervals greater than an octave, the perceived similarity of the tones depended, in part, on whether the lower frequency tone on a trial was presented first or second. In this case, the two tones were rated as more similar when the lower frequency tone was presented prior to the higher frequency tone rather than after it.

As indicated above, the group data provided no evidence of octave equivalence. However, Allen (1967) found evidence of octave equivalence only with trained musicians. For this reason, the present data were examined to determine whether those subjects who were musically trained differed in their rating responses from those subjects who were musi-

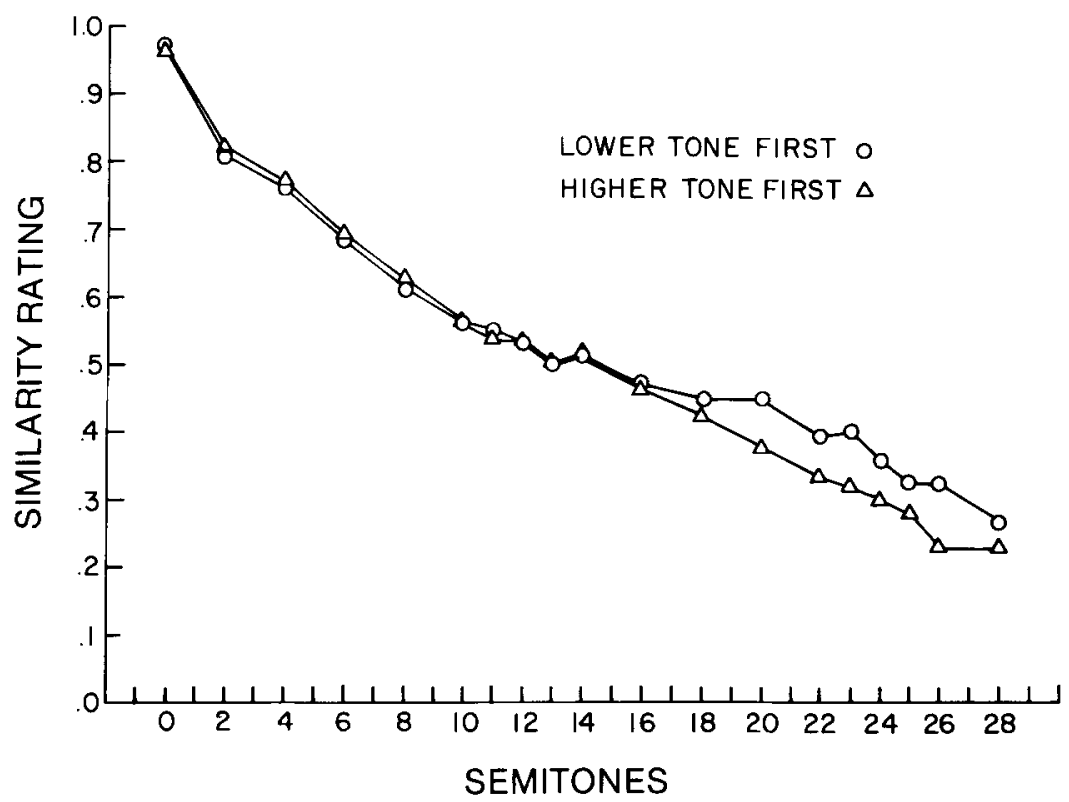

Figure 1. Mean similarity ratings in Experiment 1 as a function of the two tones' frequency separation in semitones. The curves are conditionalized on whether the firstpresented tone of the pair was the higher or lower in frequency. 


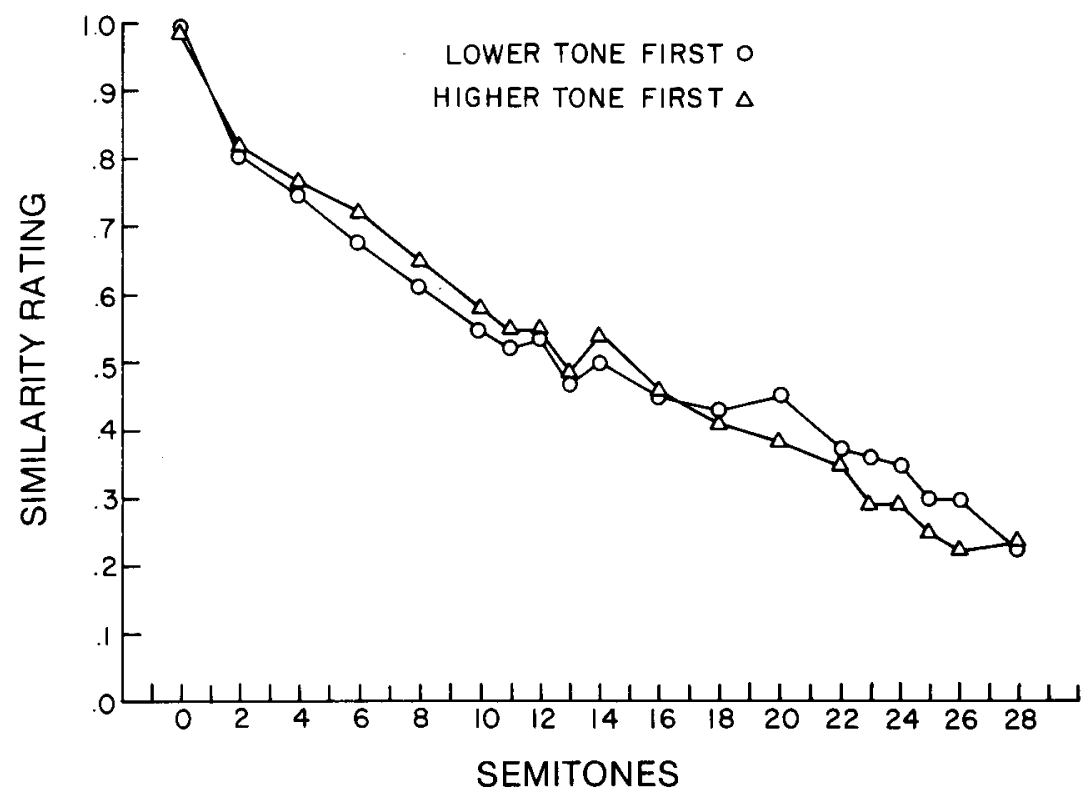

Figure 2. Mean similarity ratings of the mudically experienced subjects in Experiment 1 as a function of the two tones' frequency separntion in semitones.

cally inexperienced. For this purpose, subjects in the present experiment were divided into three groups according to their level of musical experience. Subjects in Group $1(\mathrm{~N}=14)$ had had at least 5 years of musical lessons and currently played at least one musical instrument. Subjects in Group $2(\mathrm{~N}=14)$ had taken at least 2 years of musical lessons, but did not necessarily currently play an instrument. Group 3 subjects $(\mathbf{N}=7)$ had taken less than 2 years of musical lessons. Figure 2 presents the Group 1 similarity ratings. Inspection of this figure provides little, if any, evidence of octave equivalence. There is a slight increase in the similarity ratings of tones separated by 12 semitones relative to those separated by 11 , but this increase is smaller than an unexplained increase at 14 semitones. Similarly, the ratings at 24 semitones provide little evidence of octave equivalence. The trend analysis on Sums A, B, and C, for the Group 1 data indicated a significant linear trend $[F(1,13)=$ $11.56, \mathrm{p}<.01$ ], but little evidence of a quadratic component $[F(1,13)=1.66, p>.10]$. Inspection of the data from Groups 2 and 3 indicated no evidence whatsoever of octave equivalence, and thus these data are not presented here and no trend analyses were performed.

\section{EXPERIMENT 2}

Because Allen (1967) found an effect of octave equivalence with musically trained subjects only, it was thought reasonable to replicate the experimental conditions of Experiment 1 using very highly trained musicians as subjects. The three subjects used in Ex- periment 2 were graduate students in music at the University of Wisconsin-Madison. Each had an extensive background of musical training. Because of their commitment to music, it could be assumed that the subjects in Experiment 2 were more highly trained in music than were the most highly trained subjects of Experiment 1, and the postexperimental questionnaires confirmed this. Given the high level of training, it was thought that the subjects in Experiment 2 might all demonstrate octave equivalence, at least if musical training was an important factor in obtaining this effect. The subjects were paid \$3.50 per hour for participating. The experimental procedure and design were identical to those of Experiment 1.

\section{Results}

The data for each subject were averaged over the 10 experimental blocks. Figure 3 presents the averaged similarity ratings as a function of frequency separation and direction of frequency change. The effect of frequency separation was significant $[F(18,36)$ $=13.89, \mathrm{MSe}=.026, \mathrm{p}<.01]$, but both the main effect of direction of frequency change and its interaction with frequency separation were nonsignificant $[\mathrm{F}<1, \mathrm{MSe}=.001$, and $\mathrm{F}(18,36)=1.34$, $\mathrm{MSe}=.0006$, p > .10, respectively]. Examination of the similarity ratings at intervals of 12 and 24 semitones shows an increase in rated similarity at these octave intervals relative to the adjacent nonoctave intervals. However, this effect of octave equivalence was almost exclusively the result of one of the three subject's data, as can be seen in Figure 4, which presents the individual-subject data. As Figure 4 indicates, Sub- 


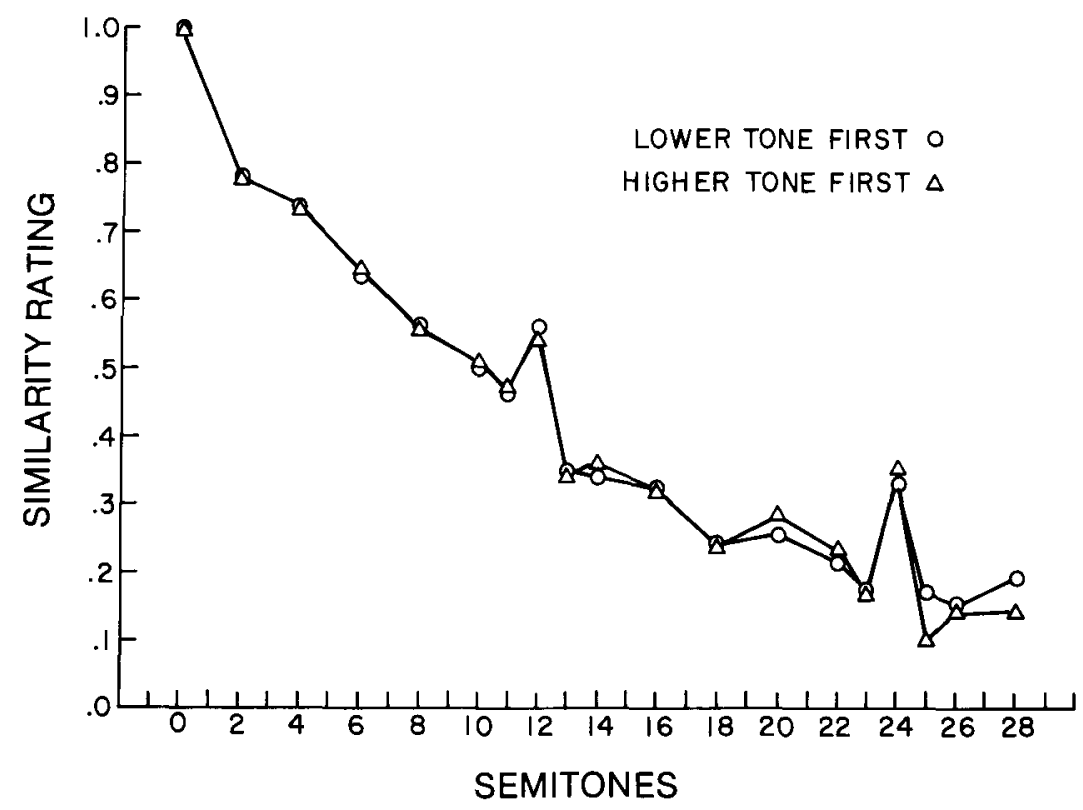

Figure 3. Mean similarity ratings of highly trained musicians in Experiment 2 as a function of the frequency difference in semitones between tones.

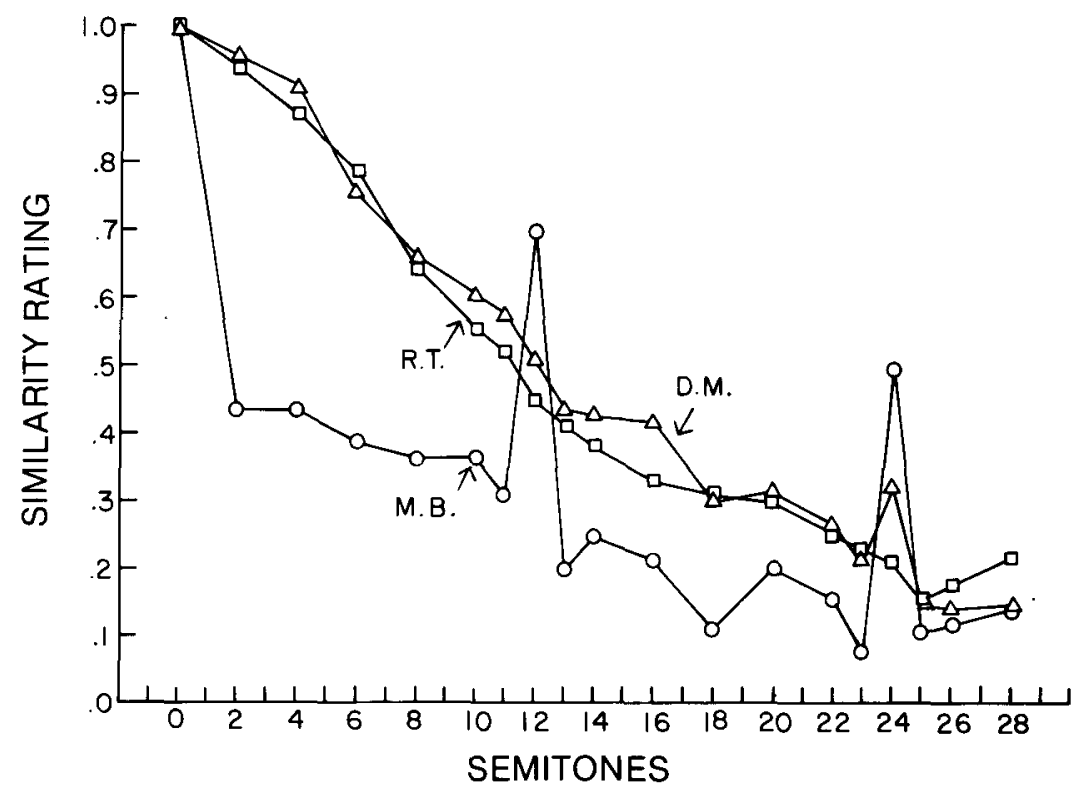

Figare 4. Similarity ratings of Individual subjects in Experiment 2 as a function of the frequency separation in semitones between tones. The three curves represent the mean ratings of the three subjects.

ject M.B. demonstrated a pronounced effect of octave equivalence, whereas Subject D.M.'s and Subject R.T.'s ratings were determined almost exclusively by the distance in semitones separating the two tones presented on a trial. Thus, the results of Experiment 2 show variability with regard to whether musicians' ratings of tone similarity are sensitive to octave-equiv- alence effects. Musical experience does not seem to guarantee a demonstration of octave equivalence.

\section{Discussion}

Experiments 1 and 2 demonstrate that octave equivalence can be an elusive phenomenon. One reason for this may be that the stimuli used were isolated 
tones; to obtain octave equivalence in a direct rating task, it might be necessary for the stimuli to be perceived as musical. Perhaps, if stimuli that are more musical were used, a musical set would be evoked and octave equivalence would clearly obtain. Experiment 3 was an attempt to test this hypothesis.

\section{EXPERIMENT 3}

On each trial of Experiment 3, subjects heard the first four notes of a familiar tune and rated how similar a distorted version of the first four notes of the tune sounded to the original. The distorted versions were identical to the original except that either the third or the fourth note was displaced upward by a variable number of semitones. Evidence for octave equivalence requires an increase in the perceived similarity of the melodies containing a 12-semitone note displacement relative to the melodies containing 11or 13-semitone displacements.

\section{Method}

Subjects. Eight students at the University of Wisconsin volunteered to earn extra points in an introductory psychology course. The subjects were from the same subject pool as in Experiment 1, although none had participated in the earlier experiment. The subjects participated in three sessions each of approximately $1 \mathrm{~h}$ duration.

Stimuli and Procedure. The subjects were first taught to recognize four familiar tunes based on hearing the first four notes of each. The four tunes were: "Frère Jacques," "Twinkle, Twinkle," "Yankee Doodle," and "Jack and Jill." Initially, the first four notes of each of these tunes were played, one tune after the other. As each tune was presented, a visual display indicated which tune it was. The notes making up each tune were presented as $60-\mathrm{msec}$ sine-wave tones with rise and fall times of $10 \mathrm{msec}$. The tones were presented binaurally at approximately $76 \mathrm{~dB}(\mathrm{~A})$, using the equipment described in Experiment 1. Ninety-five milliseconds of silence separated each note within a tune from each other. For each presentation of each tune, the frequency of the first note was determined randomly, with the restriction that the frequency be a multiple of 10 and within the range of $400-600 \mathrm{~Hz}$. Given the first tone's frequency, the frequencies of the three tones that followed were computed so as to yield the appropriate successive interval sizes for the tune under consideration. The interval sizes for each of the four tunes are presented in Table 1 .

There was a 1.5-sec silent interval between tunes. During the initial introductory part of the experiment (described above), the subjects listened to the first notes of each tune six times.

To confirm that the subjects could, indeed, identify the melodies after hearing the first four notes of each, and to further reinforce the musical set, a block of 96 identification trials followed the

Table 1

The Sequence of Pitch Changes (Intervals) in Semitones for the Four Four-Note Melodies Used in Experiments 3 and 4

\begin{tabular}{lrlr}
\hline & \multicolumn{3}{c}{ Interval } \\
\cline { 2 - 4 } & 1st & 2nd & 3rd \\
\hline Twinkle Twinkle & 0 & +7 & 0 \\
Yankee Doodle & 0 & +2 & +2 \\
Jack and Jill & -5 & +2 & -2 \\
Freres Jacques & +2 & +2 & -4 \\
\hline
\end{tabular}

introductory part of the experiment. Each trial began with the presentation of a visual warning signal for $750 \mathrm{msec}$. Two hundred and fifty milliseconds after offset of the visual warning signal, presentation of one of the four melodies began.

During the identification trials, the stimulus parameters governing the presentation of the four-note melodic sequences were identical to those used for the initial melody presentations; on each trial, the frequency of the first tone of the melodic sequence (i.e., tune) was determined randomly with the restrictions noted above. Following the presentation of the four-note melody, a visual signal (the letter $\mathbf{R}$ ), indicating the beginning of the response interval, was presented. The subjects responded by pushing one of four buttons corresponding to the four possible melodies. The response period continued until all the subjects had responded; if a subject was unsure of the correct melody s/he was instructed to guess. Two hundred and fifty milliseconds after registration of the last response, visual feedback indicating the correct response was given for $500 \mathrm{msec}$. The sequence of events constituting a new trial began $1.5 \mathrm{sec}$ after termination of the visual feedback. The four melodies were sampled randomly without replacement.

The task during the experiment proper (i.e., on those trials that followed the identification trials) was to rate on each trial how similar a distorted version of a melody seemed to an undistorted version which preceded the distorted version. A trial began with presentation of a visual warning signal for $750 \mathrm{msec}$. Two hundred and fifty milliseconds after termination of the warning signal, the appropriate (as determined by the random schedule) undistorted four-note melody was presented. The stimulus parameters governing presentation of the undistorted melodies were as outlined above. Five hundred milliseconds of silence separated offset of the last note of the undistorted four-note melodic sequence and the onset of the first note of the distorted melodic sequence. The distorted melodic sequence was identical to the undistorted sequence except that either the third or the fourth note was displaced upwards by a variable number of semitones- $0,2,4,6,8,10,11$, $12,13,14,16$, or 18 . Immediately after presentation of the distorted four-note melodic sequence, a visual signal was presented indicating the beginning of the response period. The subject's task was to rate the similarity of the distorted melodic sequence to the undistorted sequence using the rating scale described in Experiment 1 . A new trial began $1 \mathrm{sec}$ after the last subject in a group responded.

There were a total of 96 experimental conditions [number of semitones displaced (12) $\times$ melody (4) $\times$ position of note displaced (2)]; note position was a dummy variable under the 0-semitone level of number of semitones displaced. The experimental conditions occurred randomly without replacement within each block of 96 trials. A total of three blocks of 96 trials were presented on Day 1 of the experiment, although only the last two blocks were subjected to data analysis. On Day 2 , the subjects were first presented a block of 32 identification trials to refresh their memories and to reintroduce the musical set. This was followed by four blocks of 96 experimental (similarity rating) trials, all of which were included in data analysis. On Day 3, the procedure of Day 2 was repeated.

The apparatus used to deliver the stimuli was the same as in the other experiments reported above.

\section{Results}

On average, the subjects correctly identified the melodies on approximately $90 \%$ of the identification trials on Day 1 and on nearly all the identification trials on the subsequent days of the experiment. Thus, the four-note melodies were easily recognized.

The similarity rating data for each subject were averaged over the 10 experimental blocks prior to an analysis of variance. All three main effects were highly significant. First, if the third note of the melody was 
distorted, the mean similarity rating was less than when the fourth note was distorted $[F(1,7)=16.69$, $\mathrm{MSe}=.079, \mathrm{p}<.01]$ (the mean ratings were .47 and .56 , respectively). One possible explanation for this finding is that by changing the frequency of the third note, two successive intervals became distorted, whereas when the terminal (fourth) note was changed, only one successive interval became distorted. However, in a subsequent experiment using the same melodies (Experiment 5, below), this effect was not replicated.

There were differences in the mean similarity ratings of the different melodies $[\mathrm{F}(3,21)=24.87, \mathrm{MSe}=$ $.021, \mathrm{p}<.01]$, but this difference must be interpreted with reference to the significant interaction between melody and the size of the interval distortion $[\mathrm{F}(33$, 231) $=2.43$, $\mathrm{MSe}=.005, \mathrm{p}<.01$ ], which can be seen in Figure 5. Apparently, distorting the third or fourth note of "Jack and Jill" had less of an effect on the similarity ratings than did distorting the corresponding notes of the other melodies, at least when the distortion was greater than two semitones. Distorting the notes of "Twinkle, Twinkle," however, had a greater effect on the similarity ratings than did distorting the notes of the other three melodies. Although these results are potentially interesting, they were not replicated in Experiment 5 (below), and, consequently, I will provide no explanation for these findings.

The size of the note distortion (in semitones) had an effect on the similarity ratings, as can be seen in Figure $5[\mathrm{~F}(11,77)=149.34, \mathrm{MSe}=.019, \mathrm{p}<.01]$.

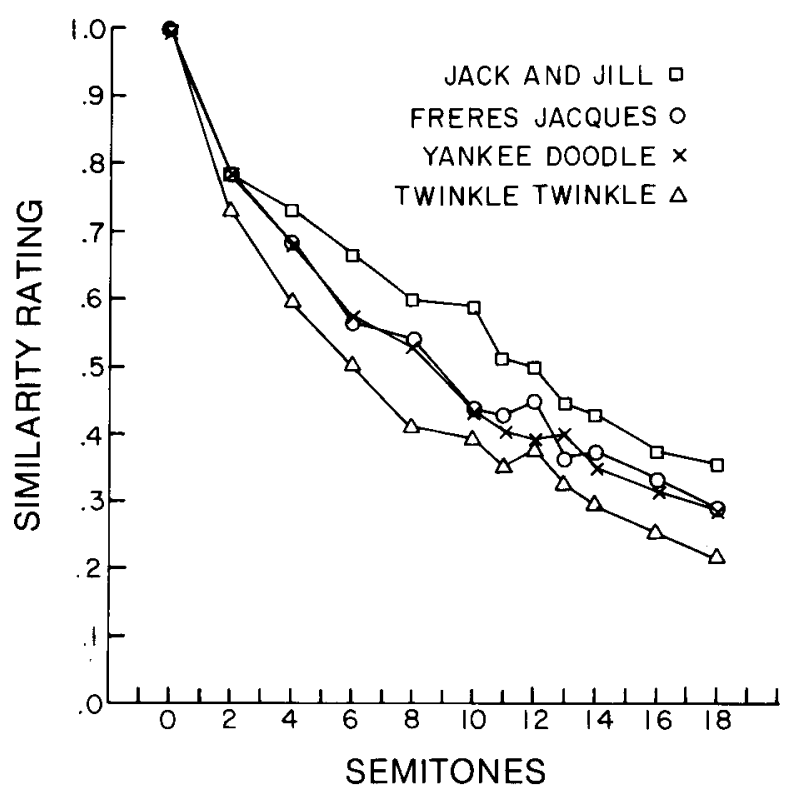

Figure 5. Mean similarity ratings of the distorted to the undistorted melodies as a function of the size in semitones of the note distortion-Experiment 3. The four curves represent the ratings of the four different melodies.

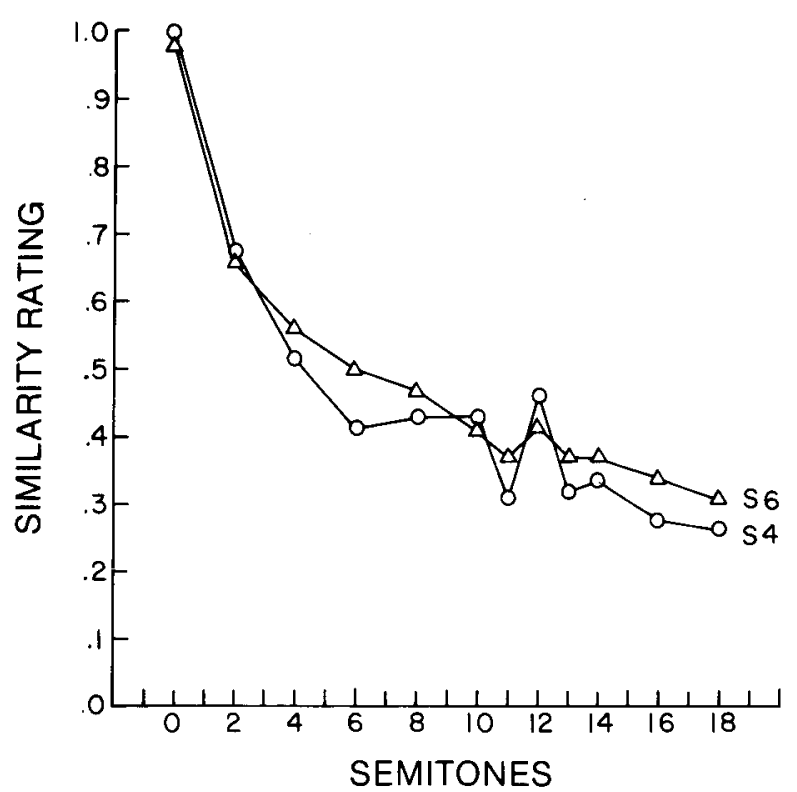

Figure 6. Mean similarity ratings (averaged over melodies) of the two subjects in Experiment 3 showing evidence of octave equivalence. The ratings are plotted as a function of the size of the distorted melodies' note distortions in semitones.

Generally, as the size of the distortion increased, the distorted melodies were rated as less similar to the originals. There appears to be some evidence of octave equivalence, at least for some of the melodies (as evidenced by a slight increase in the ratings for the 12-semitone distortions), but this effect is small and a trend analysis on the 11-, 12-, and 13-semitone data failed to provide support for a quadratic trend $[F(1,7)$ $=1.93, \mathrm{p}>.10]$ but did support a linear component $[F(1,7)=12.20, p<.01]$. Because Experiment 5 (below) provided evidence that octave equivalence is more likely to be found using the melodies "Frère Jacques" and "Twinkle, Twinkle" than the other two melodies, and because these were the two melodies in the present experiment that appeared to provide the best evidence of octave equivalence, separate trend analyses over the 11-, 12-, and 13-semitone data for these two melodies were conducted. In neither case, however, was the quadratic component significant $[F(1,7)=2.41, p>.10$, and $F(1,7)=1.16, p>.25]$ for "Frère Jacques" and "Twinkle, Twinkle," respectively.

Examination of individual-subject data revealed that only two of the subjects' data (Subjects 4 and 6) showed any evidence of octave equivalence as indexed by an increase in the mean ratings of 12-semitone distortions relative to 11 -semitone distortions. These data are presented in Figure 6. According to the criteria outlined above, Subject 4 was musically experienced and Subject 6 had an intermediate level of musical experience. Of the remaining six subjects (none of whom showed evidence of octave equivalence), four were 
musically experienced, one had an intermediate level of experience, and one was musically inexperienced.

\section{Discussion}

Experiment 3 was no more successful in providing evidence of octave equivalence than were the earlier experiments. Even given a musical context, most of the musically experienced subjects failed to show an effect of octave equivalence.

It seemed possible that the set of stimuli presented to subjects in the similarity rating experiments may have influenced the dimension(s) of the stimuli used to rate the stimuli's similarities (cf. Tversky, 1977). It is possible that absolute pitch differences dominated most of the subjects' ratings in Experiments 1-3, because the absolute frequency differences between stimuli were varied over a wide range and, consequently, may have seemed particularly salient. The limited success in finding evidence of octave equivalence may have been due to the relative salience of the absolute-pitch dimension produced by the experimental manipulations rather than to a general perceptual principle that would exclude the importance of octave equivalence. The purpose of Experiment 4 was to explore this possibility by using a relatively small range of absolute frequency differences, in so doing hopefully decreasing the salience of the absolutepitch relationships. Experiment 4 (which was modeled after Experiment 1), was undertaken to determine whether evidence of octave equivalence would be obtained when the subject's task was to rate the similarity of two tones that were separated by anywhere from 10 to 14 semitones.

\section{EXPERIMENT 4}

\section{Method}

Sabjects. Twenty-five students at the University of Wisconsin volunteered to participate in one session of approximately $1 \mathrm{~h}$. The subjects were from the same subject pool used for Experiments 1 and 3, although they did not participate in any of these earlier experiments.

Stimuli and Procedure. As in Experiment 1, the subject's task was to listen to two consecutively presented sine-wave tones and rate the degree to which they were perceived as similar to each other. The stimuli and procedure were exactly as in Experiment 1 except as noted below. The frequency separation variable had nine levels: the two tones presented on a trial were separated by from 10 through 14 semitones, in $1 / 2$-semitone increments. As in Experiment 1, whether the lower frequency tone was presented first or second on a given trial was a variable. There were, thus, a total of 18 experimental conditions. Given 18 experimental conditions, it is most reasonable to present the stimuli in blocks of 18 trials. However, due to an oversight at the time that the experiment was programmed, each trial block of the experiment consisted of 22 trials. The first 18 trials of a block consisted of a replication of the 18 different experimental conditions, and these were randomly sampled without replacement; the next 4 trials were drawn from the set of 18 possible conditions, again without replacement. Subjects participated in 15 blocks of 22 trials but only the last 14 blocks were subjected to data analysis.

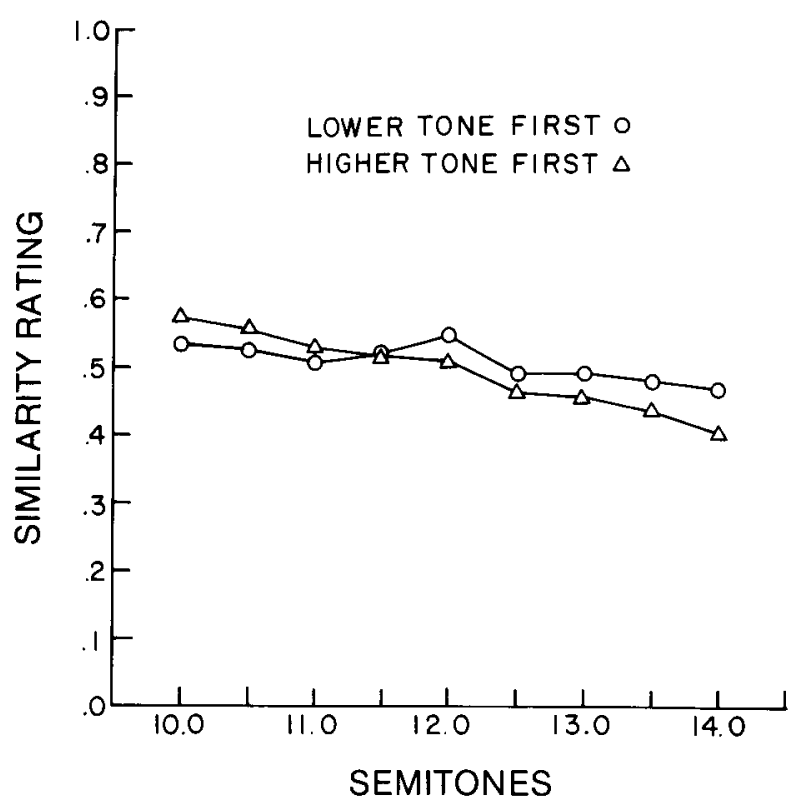

Figure 7. Mean similarity ratings in Experiment 4 as a function of the two tones' frequency separation in semitones.

\section{Results}

Figure 7 presents the mean similarity ratings as a function of the frequency difference in semitones separating the two tones on a trial; separate curves are plotted for trials in which the lower frequency tone was presented before the higher frequency tone, and vice versa. The effect of frequency separation was significant $[\mathrm{F}(8,192)=\mathbf{2 2 . 4 9}, \mathrm{MSe}=.004, \mathrm{p}<.01]$, as was the interaction between frequency separation and the temporal position of the lower frequency tone $[\mathrm{F}(8,192)=4.04, \mathrm{MSe}=.004, \mathrm{p}<.01]$.

Inspection of Figure 7 indicates an increase in the similarity ratings at the 12-semitone level of frequency separation relative to the adjacent levels of this variable. A trend analysis was performed on the data for the 11- to 13-semitone levels of frequency separation (the reason for limiting the trend analysis to this range of semitone values was to make the range of semitone values analyzed comparable to those used in the trend analyses of Experiments 1 and 3). Both the linear and quadratic components were significant $[F(1,24)=14.78, p<.01$, and $F(1,24)=4.62, p<.05$, respectively]. The significant quadratic component is of particular interest because it indicates that the effect of octave equivalence was reliable. Additional trend analyses were conducted to evaluate whether the quadratic and linear components differed as a function of whether the lower frequency tone was presented first or second on a trial. That the slope of the linear trend was greater when the higher frequency tone was presented first rather than second on a trial was confirmed by a significant interaction 
Table 2

Individual-Subject Similarity Ratings in Experiment 4 as a Function of the Frequency Differences of the Two Presented Tones

\begin{tabular}{rcccccccccc}
\hline & & \multicolumn{10}{c}{ Semitones } \\
\cline { 2 - 9 } $\mathrm{S}$ & $\mathrm{Gp}$ & 10 & 10.5 & 11 & 11.5 & 12 & 12.5 & 13 & 13.5 & 14 \\
\hline 1 & 3 & .60 & .62 & .55 & .54 & .55 & .43 & .50 & .46 & .52 \\
2 & 2 & .54 & .59 & .55 & .60 & .61 & .51 & .53 & .51 & .49 \\
3 & 2 & .53 & .42 & .41 & .36 & .40 & .30 & .24 & .22 & .22 \\
4 & 2 & .67 & .62 & .64 & .66 & .69 & .60 & .56 & .60 & .62 \\
5 & 2 & .46 & .42 & .45 & .42 & .38 & .31 & .31 & .29 & .27 \\
6 & 3 & .63 & .64 & .61 & .61 & .61 & .57 & .53 & .58 & .50 \\
7 & 2 & .54 & .50 & .54 & .42 & .38 & .41 & .43 & .40 & .33 \\
8 & 1 & .61 & .57 & .45 & .52 & .47 & .52 & .50 & .42 & .44 \\
9 & 1 & .59 & .53 & .54 & .51 & .56 & .50 & .51 & .48 & .49 \\
10 & 1 & .65 & .70 & .67 & .65 & .67 & .64 & .65 & .63 & .60 \\
11 & 3 & .54 & .62 & .51 & .38 & .48 & .37 & .43 & .34 & .43 \\
12 & 2 & .32 & .38 & .32 & .38 & .36 & .37 & .32 & .31 & .30 \\
13 & 2 & .43 & .44 & .42 & .44 & .45 & .40 & .40 & .37 & .32 \\
14 & 2 & .60 & .54 & .56 & .59 & .61 & .55 & .59 & .52 & .48 \\
15 & 2 & .57 & .65 & .50 & .59 & .63 & .47 & .59 & .53 & .58 \\
16 & 3 & .38 & .35 & .33 & .36 & .37 & .34 & .35 & .32 & .30 \\
17 & 3 & .59 & .44 & .57 & .60 & .54 & .53 & .63 & .51 & .47 \\
18 & 2 & .59 & .61 & .49 & .64 & .58 & .51 & .46 & .47 & .45 \\
19 & 1 & .50 & .51 & .50 & .51 & .50 & .51 & .48 & .49 & .47 \\
20 & 2 & .54 & .54 & .50 & .47 & .55 & .46 & .48 & .44 & .42 \\
21 & 1 & .60 & .63 & .61 & .62 & .62 & .59 & .60 & .56 & .50 \\
22 & 2 & .60 & .52 & .54 & .55 & .51 & .49 & .48 & .45 & .44 \\
23 & 1 & .64 & .59 & .60 & .60 & .61 & .52 & .43 & .50 & .39 \\
24 & 2 & .67 & .62 & .63 & .58 & .55 & .59 & .51 & .57 & .48 \\
25 & 3 & .52 & .48 & .48 & .39 & .53 & .44 & .37 & .51 & .44 \\
Mean & .555 & .541 & .519 & .519 & .528 & .477 & .475 & .458 & .437 \\
\hline
\end{tabular}

Note $-G p=$ Group, $S=$ Subject. Group $1=$ musically experienced; Group 2 =intermediate; Group $3=$ musically inexperienced.

between the linear trend component and the temporal position of the lower frequency tone $[F(1,24)=4.79$, $\mathrm{p}<.05]$. Apparently, tone height had a greater effect on the subject's ratings when the higher frequency tone was presented first. Although inspection of Figure 7 suggests that the effect of octave equivalence appeared greater when the lower frequency tone was presented first rather than second, this effect was not reliable, as the lack of a significant interaction between the quadratic trend component and the temporal position of the lower frequency tone indicates $[F(1,24)=1.22, p>.10]$.

Table 2 presents the individual-subject similarity ratings as a function of the frequency difference of the tones. The level of musical experience for each subject is also listed. Inspection of Table 2 indicates that approximately 15 of the 25 subjects demonstrated octave equivalence to some extent, as indicated by an elevation in the similarity ratings at 12 semitones. Parenthetically, note that some subjects show a gradient of equivalence centered at 12 semitones. This indicates that, for these subjects, the

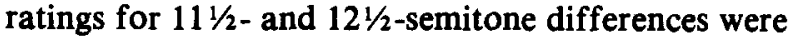
influenced by their close proximity to the octave.

\section{Discussion}

That approximately 15 of 25 subjects demonstrated an effect of octave equivalence in Experiment 4 suggests that the failure to find this level of octave equivalence in the earlier studies may have been due to the salience of the absolute-pitch dimension introduced by the wide range of absolute-pitch differences used in those experiments. However, the magnitude of the octave equivalence effect in Experiment 4 was not all that great when contrasted with the effects of pitch height differences found in the earlier experiments. Experiment 5 was an attempt to determine whether octave equivalence would obtain in greater magnitude if musical stimuli were used and the range of absolute pitch differences was comparable to those of Experiment 4.

\section{EXPERIMENT 5}

\section{Method}

Subjects. Six students at the University of Wisconsin were employed to participate in two sessions each lasting approximately $11 / 2 \mathrm{~h}$. The subjects were paid $\mathbf{\$ 3 . 5 0}$ per hour for their participation. At the time they volunteered, they knew only that the experiment was about auditory perception.

Stimuli and Procedure. The experiment was an exact replication of Experiment 3 except that the range of frequency differences used was smaller. The subjects were first taught to recognize four familiar tunes based on hearing the first four notes of each. They then participated in a block of melody identification trials. This was followed by a series of similarity rating blocks in which the subjects on each trial rated how similar a distorted version of the tune was to the earlier presented original version. The distorted version was identical to the original except that, in Experiment 5 , the third or fourth note was displaced upwards by $10,101 / 2,11$, $11 \frac{1}{2}, 12,12 \frac{1}{2}, 13,13 \frac{1}{2}$, or 14 semitones. There were a total of 72 experimental conditions [number of semitones displaced (9) $\times$ melody (4) $\times$ position of note displaced (2)], and these occurred randomly without replacement within each block of 72 trials. A total of five similarity rating blocks were presented on Day 1 (the first was considered practice), and six similarity rating blocks were presented on Day 2.

\section{Results}

The data for each subject were averaged over the 10 experimental blocks prior to an analysis of variance. The similarity ratings for each melody, as a function of the size of the interval distortion, are presented in Figure 8. The only significant effects were those of interval distortion size $[F(8,40)=2.38$, $\mathrm{MSe}=.025, \mathrm{p}<.05]$ and the interaction between melody and interval distortion size $[F(24,120)=1.84$, $\mathrm{MSe}=.007, \mathrm{p}<.05]$. An elevation in the similarity ratings occurred at approximately 12 semitones, but visual inspection indicated that the effect appeared convincing for only "Frère Jacques" and "Yankee Doodle."

A trend analysis, similar to that for Experiment 3, on the averaged data from 11-13 semitones was conducted to evaluate the linear and quadratic trend components. According to the analysis, neither the linear nor the quadratic trend was significant $[F(1,5)$ 


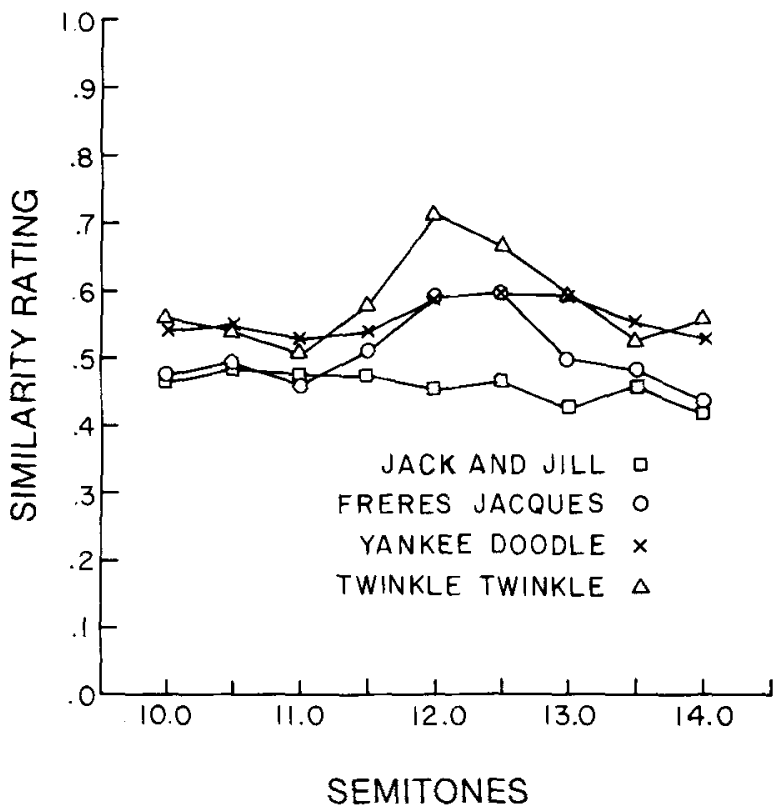

Figure 8. Mean similarity ratings of the distorted to the andistorted melodies as a function of the size in semitones of the note distortion-Experiment 5. The four curves represent the ratings of the four different melodies.

$=2.76, p>.10$, and $F(1,5)=1.62, p>.25$, respectively]. However, given the significant effect of distortion size, and the appearance of a gradient of octave equivalence centered at 12 semitones rather than an increase in rated similarity isolated to the 12 semitone data point, the trend analysis was recomputed using all nine levels of interval distortion size rather than just those from $11-13$ semitones. According to this analysis, octave equivalence was demonstrated as evidenced by a significant quadratic trend component $[F(1,5)=7.22, \mathrm{p}<.05]$; the linear component was not significant $[F(1,5)=1.45, p>.25] .^{5}$

Given the differences between melodies evident in the graph and the significant interaction between melody and size of interval distortion, separate trend analyses on the quadratic components were conducted for the individual melodies. The only melody that demonstrated a significant quadratic component was "Frère Jacques" $[F(1,5)=7.02, p<.05]$. It should be noted though that, with only six subjects, the power of the statistical tests was not great.

\section{Discussion}

Octave equivalence was demonstrated in Experiment 5, although the effect was limited. This result, in combination with the results of the earlier experiments, supports the idea that octave-equivalence effects are more likely to occur when absolute frequency differences are kept to a minimum in the experimental manipulations. In fact, in Experiment 5 there was no linear trend across frequency differ- ences, which suggests that subjects were not sensitive to the manipulation of frequency difference.

\section{GENERAL DISCUSSION}

The purpose of the present series of experiments was to determine, under a variety of conditions, whether octave equivalence would be incorporated into subjects' ratings of the perceived similarity of tones and tone sequences. The experiments failed to provide overwhelming evidence of octave equivalence. In most of the experiments, the effects of octave equivalence were small or nonexistent. In those experiments in which tone height differences between stimuli were manipulated over a wide range of values, subjects were much more sensitive to tone height manipulations than to manipulations affecting octave equivalence. However, when the manipulation of tone height differences was kept to a minimum, the effect of octave equivalence was more pronounced, although not striking. That the subjects in the different experiments focused their attention on one or another dimension of the tonal stimuli to the virtual exclusion of other available stimulus dimensions is supported by a comparison of Experiments 3 and 5. In Experiment 3, a linear decrease in the similarity ratings occurred over the range of pitch differences from 11-13 semitones, but no evidence of octave equivalence was found. In contrast, in Experiment 5 , which was identical in design to Experiment 3 except that the range of pitch differences was much smaller, no linear trend was evident in the data from 11-13 semitones, but evidence of octave equivalence was found. This tradeoff between pitch height and octave equivalence effects suggests that subjects tended to base their similarity ratings on the single most salient stimulus dimension in an experiment, or, at least, they directed most of their attention to it. This finding converges nicely with Tversky's (1977) suggestion that features of a stimulus that differentiate the stimulus from other stimuli in a similarity rating experiment will be more highly weighted in arriving at the similarity judgments than will less diagnostic features. To the extent that pitch height was varied over a wide range in the present experiments, this stimulus dimension became highly diagnostic.

Musical experience was not a good predictor of octave-equivalence effects in the present experiments. This result would appear to contrast with Krumhansl and Shepard's (1979) finding that only musicians demonstrated evidence of an hierarchy of tonal functions. On each trial in their experiments, a major scale was followed by a test tone which bore some relationship to the scale. The subject's task was to rate how well the test tone fit in with or completed the preceding major scale sequence. Only the rating responses of subjects with musical experience provided evidence of any of the musical relationships defined 
by music theory, although, even within the group of musical subjects, there was a great deal of variance with respect to the pattern of responses. In contrast to the musical subjects' data, the response patterns of musically untrained subjects was determined by absolute-pitch difference.

For musical subjects, Krumhansl and Shepard's experiment may have represented an attempt to reproduce what they had learned in their music theory classes about tonal functions; the musically untrained subjects could not have used this approach. Furthermore, Krumhansl and Shepard asked subjects, in essence, to rate a note's function within a specific musical context, a task that may have made little sense to the musically untrained. In contrast, the present experiment required subjects to rate the perceived similarity of tones or tone sequences; the only apparent "musical" factor that was evident in the judgments was octave equivalence, and both musical training and a musical context were not necessary or sufficient conditions for the demonstration of octave equivalence.

The results of the present experiments do appear to conflict with Allen's (1967) finding that musically trained subjects demonstrated octave equivalence in a tonal similarity rating task whereas untrained subjects did not. Although the reasons for this conflict are not clear, one possible explanation is that the demand characteristics of the respective experiments differed. For example, if a musician is recruited into an experiment on the basis of his/her musical training, this could affect the dimensions of the stimulus considered relevant; the similarity ratings in this case might be more reflective of musical factors than would be the case if the same subject were recruited randomly. The differences between Allen's (1967) musical and nonmusical subjects with regard to the demonstration of octave equivalence could be explained in these terms. With the exception of the three subjects of the present Experiment 2, none of the subjects in the present series were recruited on the basis of or asked about their musical experience prior to the completion of the experiment. This may explain to some extent why differences between musicians and nonmusicians were small.

Parenthetically, the similarity ratings of Experiments 1 and 4 were sensitive to whether the lower frequency tone of the pair was presented first or second. In Experiment 1, the rated similarity of two tones separated by 18 or more semitones was greater if the tone presented first was the lower in frequency. And in Experiment 4, the effect of tone height on the ratings was greater for pairs in which the higher tone was presented first. Previous research has failed to find evidence of time-error changes in pitch (Null, 1974; Postman, 1946), and thus an explanation in these terms is unlikely. However, previous research and theory have suggested that the processing of in- terval information may vary depending on whether the interval is ascending or descending (Farnsworth, 1958; Radocy, 1978; Spohn, 1965). Although the results of the present experiments are fragmentary with regard to differences between ascending and descending tone pairs, the results do suggest that this issue is worthy of additional consideration.

\section{CONCLUSIONS}

The salience of pitch height in contrast to tone chroma and octave equivalence probably depends on a number of factors, and the relevance of each characteristic to a particular experimental task may define the type of data a researcher encounters. One critical variable may be whether the experiment requires identification of the tonal stimuli or a simple rating response. As noted in the introduction, octavestretched melodies can be recognized, in some cases as well as undistorted melodies. However, this does not necessarily imply that an octave-stretched melody is perceptually equivalent to an undistorted version; subjects might actually judge them to be dissimilar. But the notes of an octave-stretched melody and those of its original do serve to identify the same melody and in this sense convey the same meaning. Analogously, a particular melody may be recognizable when played by a beginning music student, but will sound very different if performed by a professional band. The set of cues that allows identification of the tune (e.g., melodic contour, tone chroma, etc.) and thus define the common elements of the two performances may differ substantially from those cues that differentiate the performances and contribute to the judgment that the performances yield perceptually dissimilar experiences. Although octave equivalence contributes to recognition of octave-stretched melodies, the present results show that octave-equivalent tones are often not judged as perceptually very similar. Even when the octave-equivalent tones were judged as more similar to each other than were tones that were not octave equivalents, the similarity ratings indicated that octave equivalents were judged as much less similar to each other than were unisons.

Although a two-component theory of pitch may be appropriate to describe musical pitch and melody recognition, the role of octave equivalence in determining the perceived similarities of tones is less than striking. Although it is clear that octave equivalence is an important characteristic, our theories of musical perception must recognize that the equivalence of octave equivalents is clearly limited.

\section{REFERENCES}

Alten, D. Octave discriminability of musical and non-musical subjects. Psychonomic Science, 1967, 7, 421-422.

Blackwell, H. R., \& Schlosberg, H. Octave generalization, 
pitch discrimination, and loudness thresholds in the white rat. Journal of Experimental Psychology, 1943, 33, 407-419.

Deutsch, D. Music recognition. Psychological Review, 1969, 76, 300-307.

Deutsch, D. Octave generalization and tune recognition. Perception \& Psychophysics, 1972, 11, 411-412.

Deutsch, D. Octave generalization of specific interference effects in memory for tonal pitch. Perception \& Psychophysics, 1973, 13, 271-275.

DeUTsch, D. Octave generalization and melody identification. Perception \& Psychophysics, 1978, 23, 91-92.

Dowling, W. J. Musical scales and psychophysical scales: Their psychological reality. In T. Rice \& R. Falck (Eds.), Crosscultural perspectives on music. Toronto: University of Toronto Press, 1982.

Dowling, W. J., \& Hollombe, A. W. The perception of melodies distorted by splitting into several octaves: Effects of increasing proximity and melodic contour. Perception \& Psychophysics, 1977, 21, 60-64.

F ARNsworth, P. R. The social psychology of music. New York: Dryden Press, 1958.

Humphreys, L. G. Generalization as a function of method of reinforcement. Journal of Experimental Psychology, 1939, 25, 361-372.

IDson, W. L., \& Massaro, D. W. A bidimensional model of pitch in the recognition of melodies. Perception \& Psychophysics, 1978, 24, 551-565.

Kallman, H. J., \& Massaro, D. W. Tone chroma is functional in melody recognition. Perception \& Psychophysics, 1979, 26, 32-36.

Krumhansl, C. L., \& ShePard, R. N. Quantification of the hierarchy of tonal functions within a diatonic context. Journal of Experimental Psychology: Human Perception and Performance, $1979,5,579-594$.

Maggano, D. W. Kallman, H. J., \& Kelly, J. L. The role of tone height, melodic contour, and tone chroma in melody recognition. Journal of Experimental Psychology: Human Learning and Memory, 1980, 6, 77-90.

NuLL, C. H. Symmetry in judgments of musical pitch. Unpublished doctoral dissertation, Michigan State University, 1974.

Postman, L. The time-error in auditory perception. American Journal of Psychology, 1946, 59, 193-219.

RADocy, R. E. The influence of selected variables on the apparent size of successive pitch intervals. Psychology of Music, 1978, 6, 21-27.

Révész, G. An introduction to the psychology of music. Norman: Oklahoma University Press, 1954.

Spoнn, C. A new look at learning music. Instrumentalist, 1965 , 20, 62-64

Stevens, S. S., \& Galanter, E. H. Ratio scales and category scales for a dozen perceptual continua. Journal of Experimental Psychology, 1957, 54, 377-411.

Stevens, S. S., \& Volmmann, J. The relation of pitch to frequency: A revised scale. American Journal of Psychology, 1940, 53, 329-353.

Stevens, S. S., Volkmann, J., \& Newman, E. G. A scale for the measurement of the psychological magnitude of pitch. Journal of the Acoustical Society of America, 1937, 8, 185-190.

Thurlow, W. R., \& Erchul, W. P. Judged similarity in pitch of octave multiples. Perception \& Psychophysics, 1977, 22, 177-182.

Tversky, A. Features of similarity. Psychological Review, 1977, 84, 327-352.

\section{NOTES}

1. Throughout the present article, I will adopt Révész's use of the term equivalent note, and I will refer to notes that share a common tone chroma but occur in different octaves as octave equivalents. However, it should be stressed that the term implies that the notes are equivalent only in terms of tone chroma and not tone height.

2. Deutsch $(1969,1972,1978)$ has argued that the substitution of octave-equivalent notes in a melody results in a decrease in recognition to chance levels. She assumes that although single tones may be analyzed for tone chroma, melody recognition involves the direct abstraction of the absolute sizes of successive intervals without reference to chroma information. To support the absence of chroma utilization in melody recognition, Deutsch (1972) reported an experiment in which the notes of the tune "Yankee Doodle" were each randomly displaced into one of three contiguous octaves but the original chroma was preserved; in this case, recognition of the tune was poor. Deutsch argued that if octave equivalence operated in melody recognition, subjects should have had little difficulty in recognizing the tune. However, a number of researchers have challenged Deutsch's interpretation of her results (Dowling \& Hollombe, 1977; Idson \& Massaro, 1978; Kallman \& Massaro, 1979; Massaro, Kallman, \& Kelly, 1980) because, by randomly displacing the notes into one of three contiguous octaves, Deutsch destroyed the original melodic contour of the tune. It has since been shown that it was the violation of melodic contour rather than a failure to use chroma information that resulted in the poor recognition performance in Deutsch's experiment. The evidence now suggests that, contrary to Deutsch's claims, chroma information is used in the recognition of both familiar and unfamiliar melodies. Furthermore, this result obtains for familiar melodies irrespective of whether subjects are told in advance the set of melodies that they will be asked to recognize (Kallman \& Massaro, 1979).

3. A semitone difference is equivalent to moving from one piano key to an adjacent key. Two notes are said to be separated by a semitone if the ratio of their fundamental frequencies is approximately 1.0595:1.

4. Because octave equivalents share common tone chroma but differ in pitch height, the demonstration of octave equivalence requires only that octave-equivalent notes be rated as more similar to one another than notes that do not share a common tone chroma. Because octave equivalents differ in pitch height, it is expected that unison notes (which are identical in both chroma and pitch height) should be judged as more similar to each other than are octave equivalents.

5. Given that octave equivalence effects were found in melody recognition experiments only when the melodic contour remained undistorted (Dowling \& Hollombe, 1977; Idson \& Massaro, 1978; Kallman \& Massaro, 1979; Massaro, Kallman, \& Kelly, 1980), it is informative to determine whether octave equivalence was more likely to obtain in the present Experiment 5 when the interval distortion of the comparison tune left the melodic contour intact. For example, the contour of the first three intervals of "Frère Jacques" may be represented as + + - ("+ " represents an ascending interval, "-" a descending interval). If the third note of the tune is displaced upwards by between 10 and 14 semitones, the melodic contour of the tune does not change from the original. However, if the fourth note is displaced upwards by 10-14 semitones, the direction of pitch change for the last successive interval changes from negative to positive and, as a result, the original melodic contour is violated.

Examination of the data from Experiment 5 indicated that the octave-equivalence effect did not differ as a function of whether the comparison melody maintained the original contour or not; octave equivalence was demonstrated in both cases. At first glance, this result might seem to contradict the earlier findings that octaveequivalence effects are evident only when the contour of the original melody is maintained, but there are good reasons to question whether the disparate results indicate a contradiction. Deutsch $(1972,1978)$ suggested that the cues necessary to recognize a melady may differ, depending on whether or not the subject has a hypothesis about the identity of the melody. For example, if the subject entertains the hypothesis that a melody is "Yankee Doodle," the subject could imagine (construct auditory images of) the notes of the tune and, as they are played, monitor whether the played 
notes match the chroma of the hypothesized tune. Such a strategy might be insensitive to contour violations because the subject could perform the task simply by making a chroma match on each note. The present experiment offered a perfect opportunity for the use of such a strategy, because the subjects heard the notes of the undistorted melody just prior to presentation of the distorted melody and thus could have maintained auditory images of the undistorted melody's notes for comparison. In contrast, when a subject must recognize a melody from an unspecified set of alternatives, the chroma-testing strategy is inadequate and correct contour, as well as chroma information, is necessary for recognition (Kallman \& Massaro, 1979).

(Manuscript received October 26, 1981; revision accepted for publication March 25, 1982.) 